JOURNAL OF THE

CHUNGCHEONG MATHEMATICAL SOCIETY

Volume 27, No. 1, 2014

http://dx.doi.org/10.14403/jcms.2014.27.1.145

\title{
PORTFOLIO SELECTION WITH NONNEGATIVE WEALTH CONSTRAINTS: A DYNAMIC PROGRAMMING APPROACH
}

\author{
YONG HYUn SHIN*
}

\begin{abstract}
I consider the optimal consumption and portfolio selection problem with nonnegative wealth constraints using the dynamic programming approach. I use the constant relative risk aversion (CRRA) utility function and disutility to derive the closed-form solutions.
\end{abstract}

\section{Introduction}

Following the seminal works of Merton [9, 10], there have been many research articles on the continuous-time portfolio optimization problems with various economic constraints. Especially nonnegative wealth constraint, which is sometimes called borrowing constraint, is one of the most important economic constraints on portfolio selection problems. The nonnegative wealth constraint means that the agent cannot borrow against her future labor income, that is, she always supports a nonnegative wealth level (refer to $[1,2,3,4,5,7,8]$ ).

In this paper I investigate the optimal consumption and portfolio selection problem with nonnegative wealth constraints based on the dynamic programming framework of Karatzas et al. [6]. I use the constant relative risk aversion (CRRA) utility function and disutility to obtain the closed-form solutions.

\section{The economy}

It is assumed that only two assets are traded in the financial market. One is a riskless asset and the other is a risky asset. The constant interest

Received January 08, 2014; Accepted January 27, 2014.

2010 Mathematics Subject Classification: Primary 91G10.

Key words and phrases: nonnegative wealth constraints, dynamic programming approach, CRRA utility, portfolio selection. 
rate is $r>0$, and the risky asset follows the geometric Brownian motion $d S_{t} / S_{t}=\mu d t+\sigma d B_{t}$, where $\mu$ and $\sigma$ are constants, and $B_{t}$ is a standard Brownian motion on a probability space $(\Omega, \mathcal{F}, \mathbb{P})$.

The market price of risk is defined by $\theta:=(\mu-r) / \sigma$. Let $\pi_{t}$ be the $\mathcal{F}_{t}$-progressively measurable portfolio process at time $t$ and $c_{t}$ be the nonnegative $\mathcal{F}_{t}$-progressively measurable consumption rate process at time $t$. It is assumed that the following mathematical conditions hold:

$\int_{0}^{t} c_{s} d s<\infty$ and $\int_{0}^{t} \pi_{s}^{2} d s<\infty$, for all $t \geq 0$, almost surely (a.s.).

The agent receives constant labor income $y_{0}>0$. So the agent's wealth process $X_{t}$ at time $t$ follows the stochastic differential equation (SDE)

$$
d X_{t}=\left[r X_{t}+\pi_{t}(\mu-r)-c_{t}+y_{0}\right] d t+\sigma \pi_{t} d B_{t}, X_{0}=x \geq 0 .
$$

It is assumed that the agent in this model always faces nonnegative wealth constraints such that

$$
X_{t} \geq 0, \text { for all } t \geq 0 .
$$

\section{The optimization problem}

The agent's optimization problem is to maximize her expected utility

$$
V(x)=\sup _{(c, \pi) \in \mathcal{A}(x)} \mathbb{E}\left[\int_{0}^{\infty} e^{-\rho t}\left(\frac{c_{t}^{1-\gamma}}{1-\gamma}-l\right) d t\right],
$$

where $\gamma>0(\gamma \neq 1)$ is the agent's coefficient of relative risk aversion, $\rho>0$ is a subjective discount rate, $l>0$ is constant disutility due to labor, and $\mathcal{A}(x)$ is an admissible set of pairs $(c, \pi)$. The following assumption always holds without further comments:

Assumption 3.1.

$$
K:=r+\frac{\rho-r}{\gamma}+\frac{\gamma-1}{2 \gamma^{2}} \theta^{2}>0
$$

REMARK 3.2. For later use, I consider a quadratic equation

$$
\frac{1}{2} \theta^{2} m^{2}+\left(\rho-r+\frac{1}{2} \theta^{2}\right) m-r=0,
$$

with two real roots $m_{+}>0$ and $m_{-}<-1$.

Next theorem implies my main results. 
THEOREM 3.3. The value function of this optimization problem (3.1) is given by

$$
V(x)=\frac{r-\frac{1}{2} \theta^{2} m_{+}}{\rho} D_{1} \xi^{-\gamma m_{+}-\gamma}+\frac{1}{(1-\gamma) K} \xi^{1-\gamma}-\frac{l}{\rho}, \text { for } x \geq 0,
$$

where

$$
\hat{c}=\frac{\gamma m_{+}}{\gamma m_{+}+1} \frac{K y_{0}}{r}>0, \quad D_{1}=\frac{1}{r\left(\gamma m_{+}+1\right)} y_{0} \hat{c}^{\gamma m_{+}}>0,
$$

and $\xi$ is the solution to the algebraic equation

$$
x=D_{1} \xi^{-\gamma m_{+}}+\frac{\xi}{K}-\frac{y_{0}}{r} .
$$

And the optimal policies are given by $\left(c^{*}, \pi^{*}\right)$ such that

$$
c^{*}=\xi \quad \text { and } \quad \pi^{*}=\frac{\theta}{\sigma \gamma}\left(-\gamma m_{+} D_{1} \xi^{-\gamma m_{+}}+\frac{\xi}{K}\right), \text { for } x \geq 0 .
$$

Proof. From the optimization problem (3.1), the Bellman equation is derived as follows:

$$
\begin{aligned}
\max _{(c, \pi)} & {\left[\left\{r x+\pi(\mu-r)-c+y_{0}\right\} V^{\prime}(x)\right.} \\
+ & \left.\frac{1}{2} \sigma^{2} \pi^{2} V^{\prime \prime}(x)-\rho V(x)+\frac{c^{1-\gamma}}{1-\gamma}-l\right]=0 .
\end{aligned}
$$

The first-order conditions (FOCs) of the Bellman equation (3.2) yield

$$
c=\left\{V^{\prime}(x)\right\}^{-\frac{1}{\gamma}} \quad \text { and } \quad \pi=-\frac{\theta V^{\prime}(x)}{\sigma V^{\prime \prime}(x)} .
$$

Substituting the FOCs (3.3) into the Bellman equation (3.2), then I obtain

$$
r x V^{\prime}(x)+y_{0} V^{\prime}(x)-\frac{1}{2} \frac{\theta^{2}\left\{V^{\prime}(x)\right\}^{2}}{V^{\prime \prime}(x)}+\frac{\gamma}{1-\gamma}\left\{V^{\prime}(x)\right\}^{-\frac{1-\gamma}{\gamma}}-\rho V(x)-l=0 .
$$

It is assumed that the optimal consumption $c^{*}=C(x)$ is considered as a function of wealth and that $X(\cdot)$ is the inverse function of $C(\cdot)$. Then the FOCs (3.3) give

$$
V^{\prime}(x)=C(x)^{-\gamma} \quad \text { and } \quad V^{\prime \prime}(x)=\frac{-\gamma C(x)^{-\gamma-1}}{X^{\prime}(c)} .
$$

Plugging (3.5) into the changed Bellman equation (3.4), then I obtain (3.6) $r c^{-\gamma} X(c)+y_{0} c^{-\gamma}+\frac{\theta^{2}}{2 \gamma} c^{1-\gamma} X^{\prime}(c)+\frac{\gamma}{1-\gamma} c^{1-\gamma}-\rho V(X(c))-l=0$. 
Taking derivative of the equation (3.6) with respect to $c$, then I have

$$
\begin{aligned}
& r c^{-\gamma} X^{\prime}(c)-r \gamma c^{-\gamma-1} X(c)-\gamma y_{0} c^{-\gamma-1}+\frac{\theta^{2}}{2 \gamma} c^{1-\gamma} X^{\prime \prime}(c) \\
& +\frac{(1-\gamma) \theta^{2}}{2 \gamma} c^{-\gamma} X^{\prime}(c)+\gamma c^{-\gamma}-\rho c^{-\gamma} X^{\prime}(c)=0 .
\end{aligned}
$$

Thus I derive the second order ordinary differential equation (ODE)

$$
\frac{\theta^{2}}{2 \gamma} c^{2} X^{\prime \prime}(c)+\left(r-\rho+\frac{(1-\gamma) \theta^{2}}{2 \gamma}\right) c X^{\prime}(c)-r \gamma X(c)+\gamma c-\gamma y_{0}=0
$$

From ODE (3.7), I conjecture the solution $X(c)$ of the form

$$
X(c)=D_{1} c^{-\gamma m_{+}}+\frac{c}{K}-\frac{y_{0}}{r},
$$

for some constant $D_{1}$. Then there exists a consumption level $\hat{c}>0$ such that

$$
X(\hat{c})=0 \quad \text { and } \quad X^{\prime}(\hat{c})=0
$$

because of the nonnegative wealth constraint (2.1). From (3.9), I can determine $\hat{c}$ and $D_{1}$. Thus I obtain

$$
\hat{c}=\frac{\gamma m_{+}}{\gamma m_{+}+1} \frac{K y_{0}}{r}>0 \quad \text { and } \quad D_{1}=\frac{1}{r\left(\gamma m_{+}+1\right)} y_{0} \hat{c}^{\gamma m_{+}}>0 .
$$

I can easily check that $X(c)$ in (3.8) is an increasing function for $c \in$ $(\hat{c}, \infty)$ since

$$
X^{\prime}(c)=-\gamma m_{+} D_{1} c^{-\gamma m_{+}-1}+\frac{1}{K}>-\gamma m_{+} D_{1} \hat{c}^{-\gamma m_{+}-1}+\frac{1}{K}=0,
$$

for $c \in(\hat{c}, \infty)$. Now plugging $X(c)$ in (3.8) into (3.6), then I obtain the value function

$$
V(x)=\frac{r-\frac{1}{2} \theta^{2} m_{+}}{\rho} D_{1} \xi^{-\gamma m_{+}-\gamma}+\frac{1}{(1-\gamma) K} \xi^{1-\gamma}-\frac{l}{\rho},
$$

where $\xi$ is the solution to the algebraic equation

$$
x=D_{1} \xi^{-\gamma m_{+}}+\frac{\xi}{K}-\frac{y_{0}}{r} .
$$

Also I use the FOCs (3.3) and (3.5) with the value function $V(x)$ in (3.10) to obtain the optimal policies $\left(c^{*}, \pi^{*}\right)$. 


\section{References}

[1] K. J. Choi, G. Shim, and Y. H. Shin, Optimal Portfolio, Consumption-Leisure and Retirement Choice Problem with CES Utility, Math. Finance 18 (2008), 445-472.

[2] P. H. Dybvig and H. Liu, Lifetime Consumption and Investment: Retirement and Constrained Borrowing, J. Econ. Theory 145 (2010), 885-907.

[3] N. El Karoui and M. Jeanblanc-Picquè, Optimization of Consumption with Labor Income, Finance Stochast. 2 (1998), 409-440.

[4] E. Farhi and S. Panageas, Saving and Investing for Early Retirement: A Theoretical Analysis, J. Financial Econ. 83 (2007), 87-121.

[5] H. He and H. F. Pages̀, Labor Income, Borrowing Constraints, and Equilibrium Asset Prices, Econ. Theory 3 (1993), 663-696.

[6] I. Karatzas, J. P. Lehoczky, S. P. Sethi, and S. E. Shreve, Explicit Solution of a General Consumption/Investment Problem, Math. Oper. Res. 11 (1986), 261-294.

[7] H. K. Koo, Consumption and Portfolio Selection with Labor Income: A Continuous Time Approach, Math. Finance 8 (1998), 49-65.

[8] B. H. Lim and Y. H. Shin, Optimal Investment, Consumption and Retirement Decision with Disutility and Borrowing Constraints, Quant. Finance 11 (2011), 1581-1592.

[9] R. C. Merton, Lifetime Portfolio Selection under Uncertainty: The ContinuousTime Case, Rev. Econ. Stat. 51 (1969), 247-257.

[10] R. C. Merton, Optimum Consumption and Portfolio Rules in a ContinuousTime Model, J. Econ. Theory 3 (1971), 373-413.

Department of Mathematics

Sookmyung Women's University

Seoul 140-742, Republic of Korea

E-mail: yhshin@sookmyung.ac.kr 